\title{
LETTER
}

\section{Quantitative partitioning of regional and local processes shaping regional diversity patterns}

Tara K. Rajaniemi, ${ }^{1 *}$ Deborah E. Goldberg, $^{2}$ Roy Turkington ${ }^{3}$ and Andrew R. Dyer ${ }^{2}$

${ }^{1}$ Department of Biology, Indiana University, Bloomington, IN 47405, USA ${ }^{2}$ Department of Ecology and Evolutionary Biology, University of Michigan, Ann Arbor, MI 48109, USA

${ }^{3}$ Department of Botany and Biodiversity Research Center, University of British Columbia, Vancouver, BC V6T 1Z4, Canada *Correspondence and Present address: Biology Department, University of Massachusetts Dartmouth, North Dartmouth, MA 02747, USA. E-mail: trajaniemi@umassd.edu Present address: Andrew $R$. Dyer, Department of Biology and Geology, University of South Carolina-Aiken, Aiken, SC 29801, USA

\begin{abstract}
Much recent research explaining plant community diversity has focused on comparing the relative impacts of regional and local processes. We employed a novel analysis to quantify the effects of multiple regional and local processes on species richness, and to make quantitative comparisons of those effects across two sites that differ in plot-level species richness, productivity and environmental conditions. While abiotic stress and competition limited richness within the communities at both sites, only differences in the overall pool of species at the site, likely resulting from long-distance dispersal and climate fluctuations, explained the differences in plot-level richness between sites. Patterns in local richness may be driven by a temporal storage effect, with greater richness in the site with greater climatic variability. By identifying both the factors that impact diversity within communities and those that vary systematically across communities, our integrated approach provides a better understanding of regional diversity patterns.
\end{abstract}

\section{Keywords}

Local vs. regional effects, productivity-diversity patterns, regional diversity patterns, species pools, species richness, temporal storage effect.

\section{INTRODUCTION}

Much recent research in explaining plant community diversity has focused on assessing the relative importance of regional processes, such as speciation and large-scale migration, and local processes, such as abiotic stress and competition (e.g. Zobel 1992, 1997; Ricklefs 2004). Many studies show that regional and local processes both have large impacts on species diversity, and considering multiple processes simultaneously has greatly improved our ability to explain variation in local diversity (Grace 2001). Two distinct approaches have dominated contributions to our understanding of the importance of local and regional processes to date.

Experimental approaches to this question have focused on one key regional influence on diversity, dispersal from the regional species pool, and have examined how the importance of the species pool varies along local environ- mental gradients. Typically, seeds are added to plots, and if seed addition increases diversity, diversity is considered to be limited by dispersal from the species pool. Such studies have shown that the importance of the species pool varies with local conditions including the diversity and composition of existing species (Tilman 1997), intensity of competition (Xiong et al. 2003; Mouquet et al. 2004), disturbance (Xiong et al. 2003; Foster et al. 2004; but see Zobel et al. 2000) and productivity (Foster \& Dickson 2004). This approach can also reveal complex interactions, e.g. disturbance may interact with species pool effects more in productive (e.g. Xiong et al. 2003; Foster et al. 2004) than unproductive sites (e.g. Zobel et al. 2000; Foster et al. 2004).

Other studies use statistical approaches to address the question of multiple influences on diversity. Several authors have used multiple regression (Gough et al. 1994; Safford et al. 2001) or structural equation modelling (Grace \& Pugesek 1997; Grace \& Jutila 1999; Weiher 2003; Weiher 
et al. 2004) to explain variation in diversity across a set of sites. Structural equation models explain observed diversity in terms of direct effects of abiotic conditions and/or disturbance (their effects on the local species pool), direct effects of species interactions, and indirect effects of abiotic conditions via their effects on interactions. These studies generally find that variation in diversity is best described by a combination of factors (Grace 2001). For instance, in coastal marshes, local richness depends primarily on direct and indirect effects of abiotic conditions, with smaller, indirect effects of disturbance (Grace \& Pugesek 1997). In oak savanna understory, disturbance and shading by the tree canopy have strong, positive effects on the species pool, and soil conditions influence richness by both direct effects on the species pool and indirect effects via species interactions (Weiher 2003).

Both these approaches have shown that species pools can influence richness. However, they have not been used to quantify contributions of different pools and the ecological processes that determine the composition of each pool. Species pools can be defined at a range of scales. For example, Partel et al. (1996) suggested three levels of species pools: (i) the regional pool, the set of species occurring in the region; (ii) the local pool, the set of species occurring in the local landscape; and (iii) the actual (or community) pool, the species coexisting in the target community. Migration and dispersal, abiotic factors and biotic interactions filter species from each pool to create the next, smaller pool (Keddy 1992; Zobel 1997; Zobel et al. 1998). In this paper, we use a filter model approach to quantify the magnitude of effects of multiple local and regional processes on plot-level species richness in two sites that differ in a number of important environmental variables, including rainfall, temperature and productivity. Starting with a regional pool composed of all species observed at both sites over multiple years, we identify multiple, nested species pools at successively smaller spatial and/or temporal scales and the ecological processes that filter (remove) species from each larger scale pool to the smaller-scale pool. We then quantify the effects of each filter on species richness in the pool as the deviation of actual richness from that expected if the filter did not exist and compare these between sites (Fig. 1). To assess the role of different ecological processes at each filter, we then relate the magnitude of this deviation to measures of the abiotic and biotic environment. This stepwise assessment allows us to determine to what extent local richness in a single community is shaped by the regional species pool, site-level environment and neighbourhood (plot-level) interactions. Furthermore, comparing the parallel analyses in two sites allows us to determine to what extent changes in the effects of the regional species pool, local environment and/or neighbourhood interactions can account for regional patterns in species richness.

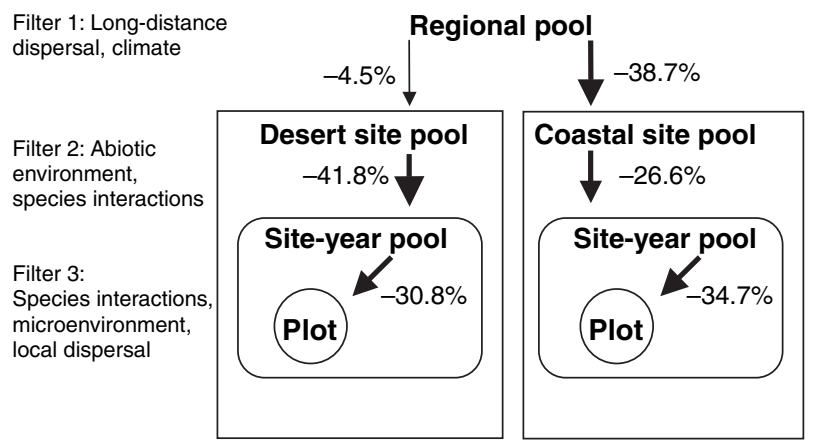

Figure 1 Summary of filter model, indicating hierarchical pools and the filters eliminating species from each. Thickness of arrows indicates relative impacts of each filter in our system. Values are percent deviation of observed species richness from that expected if only sample size, not the ecological filter, determines pool richness. Average deviations over five site-year pools per site and over all plots in a site are shown.

We are thus testing for correlates of the amount of filtering between pools, rather than correlates of observed richness in any given pool, with the ultimate goal of understanding the filters and ecological processes that determine richness in each of these pools. The magnitude of any filter is a direct reflection of the beta diversity among pools at the smaller-scale - if no species were removed by a filter, all smaller-scale pools would be identical to each other and to the larger-scale pool in which they are nested.

\section{METHODS}

\section{Vegetation surveys}

We used data from 5 years of vegetation surveys of annual plant communities on semi-stabilized sand dunes in Israel. We studied two sites $200 \mathrm{~km}$ apart, a coastal site (Caesarea; $32^{\circ} 30^{\prime} \mathrm{N}, 34^{\circ} 55^{\prime} \mathrm{E}$ ) and a desert site (Holot Mashabim; $\left.31^{\circ} 00^{\prime} \mathrm{N}, 34^{\circ} 44^{\prime} \mathrm{E}\right)$. Both have a Mediterranean climate with a winter growing season, but the coastal site has higher rainfall, more moderate temperatures, greater annual plant production, and in most years, lower species density than the desert site (Table 1).

Vegetation was sampled from each of the sites in late March to early April in each of 5 years. In 1993, 1994 and 1995 a total of 36 samples were collected from random intervals along three, 12-m-long transects in each site in each year. Plot sizes were $144 \mathrm{~cm}^{2}$ in 1993 and 1994, and $625 \mathrm{~cm}^{2}$ in 1995. In 1997, 11 samples, each $0.32 \mathrm{~m}^{2}$, were collected from each site. In 1998, 20 plots of $0.16 \mathrm{~m}^{2}$ and two to five plots of $0.32 \mathrm{~m}^{2}$ were sampled in each site. No plants were sampled in 1996 because rainfall began too late in the growing season for seeds to germinate at the desert site. Consequently, no annual plants grew at that site in that 
Table 1 Environmental and plant community characteristics of the two study sites

\begin{tabular}{|c|c|c|c|}
\hline & Coastal site & Desert site & $P$-value \\
\hline \multicolumn{4}{|c|}{ Annual precipitation (1 October to 31 September) (mm) } \\
\hline Long-term average & 550 & 110 & \\
\hline 5 study years & 518 & 97 & $* * *$ \\
\hline \multicolumn{4}{|c|}{ Temperature during growing season $\left({ }^{\circ} \mathrm{C}\right)$} \\
\hline Mean & 20.6 & 20.1 & n.s. \\
\hline Minimum & 4.3 & 3.1 & n.s. \\
\hline Maximum & 38.2 & 41.0 & $*$ \\
\hline Standing crop $\left(\mathrm{g} \mathrm{m}^{-2}\right)$ & 261.6 & 66.1 & ** \\
\hline \multicolumn{4}{|c|}{ Species richness, mean per plot } \\
\hline 1993 & 6.69 & 5.52 & n.s. \\
\hline 1994 & 5.93 & 5.83 & n.s. \\
\hline 1995 & 11.05 & 15.78 & $* * *$ \\
\hline 1997 & 14.64 & 21.91 & $* * *$ \\
\hline 1998 & 11.63 & 20.20 & $* * *$ \\
\hline
\end{tabular}

Significance is based on one-way ANOVA with $n=10$ (one-point per site per year) for precipitation, temperatures and standing crop. $P$-values for species richness are for one-way AnCOVA on species richness in each plot, with shoot number as a covariate. Plot sizes were small $\left(144 \mathrm{~cm}^{2}\right)$ in 1993 and 1994, resulting in low mean richness and non-significant differences between sites; 1998 values are for the $0.16 \mathrm{~m}^{2}$ plots only.

n.s., not significant; $* P<0.05,{ }^{* *} P<0.01,{ }^{* * *} P<0.001$.

year, and the other site was not sampled. Although the sampling in different years involved variable plot numbers and sizes, and therefore the number of plants sampled each year differed, our analyses (below) use random sampling to remove effects of sample size. Therefore, the variation in sampling methods should not affect our conclusions.

In each plot, plants were dug up and immediately frozen. Samples were subsequently defrosted, sorted to species, individuals counted, and aboveground mass weighed. The total number of individuals (species) sampled over the 5 years was $25606(127)$ in the desert site and 31256 (84) in the coastal site.

Data on daily rainfall and temperatures for the two sites were obtained from the Israel Meteorological Service (Bet Dagan, Israel).

\section{Analysis}

We identified four nested levels of species pools, with three categories of processes acting on them (Fig. 1). Pools contain information about both the number of species and the abundance of each; therefore, we refer to pools as comprising individual plants rather than a simple species list.

We consider the sites to be drawing species from a single 'regional' pool that includes all plants observed across all years and both sites (total of 56862 individuals in 164 species). A regional pool for a location should, in principle, include all species capable of reaching that location. Many species are clearly able to reach both our sites, confirming that they belong to a single regional pool. The sites are located on the edge of several biogeographical provinces and so represent an overlapping mix of geographical affinities (Zohary 1966). Furthermore, of the species found in these vegetation surveys and in garden experiments using seed bank from the two sites (Goldberg \& Estabrook 1998; Goldberg et al. 2001), 68\% of the species found in the desert were also present at the coastal site, and $81 \%$ of the species found at the coastal site were also found at the desert site. Of course, many species potentially capable of reaching the sites were not observed in the vegetation surveys and therefore were not part of our regional pool. Such species are not easily incorporated into the pool, as we have no way to determine their appropriate relative abundance in the pool. Failing to include these species in the pool decreases the apparent difference between the regional and site pools, but does so equally for each site, and so should not affect any between-site comparisons.

Lack of long-distance dispersal or inappropriate climate at each site filters species from the regional pool to create a 'site' pool, including all plants observed across all years at a single site. Substrate was almost pure sand with similar texture at both sites, although the coastal site was slightly more organic; therefore, substrate was not expected to be an important source of variation between sites. Abiotic conditions and species interactions filter each site pool to the 'site-year' pool (all plants observed at that site in a given year). The conditions prevailing in a given year (e.g. rainfall and temperature) may be too harsh to allow a species to survive in that year, or it may be eliminated by competitive exclusion if conditions contribute to intense interactions throughout the site in that year. Finally, species interactions, local dispersal and variation in microenvironment filter the site-year pool to produce the species composition of a single 'plot'.

The numbers of species in the various pools cannot be directly compared, because they are based on different sample sizes (number of individuals). Therefore, we used random sampling from a given pool to calculate the expected species richness of a sample of the size of the next nested pool if no filters were operating. Random sampling was from a pool consisting of all species observed in the indicated set of plots, with their abundances (number of shoots collected). The probability of selecting a species from the pool was proportional to its relative abundance in the pool. Each calculation of expected richness is based on the mean of 500 random samples, with total plant number in each sample equal to the number observed in the next nested pool. We quantified the effects of ecological filters on species richness as the deviation of observed richness 
from that expected given random sampling, expressed as a $\log$ response ratio

$\ln \mathrm{RR}=\ln \left(\frac{\text { observed richness }}{\text { expected richness }}\right)$.

Reported $P$-values for this deviation are the proportion of random samples in which richness was less than or equal to richness observed in the next nested pool.

To assess the roles of different ecological processes, we used regression and ANCOVA to test for effects of yearly rainfall (site to site-year filter), plant production (site to site-year filter); mean, minimum and maximum temperature (site to site-year filter); and plant density within plots (site-year to plot filter) on deviation from expected richness.

\section{RESULTS}

The first filter, from regional to site pools, reduced species richness at both sites, but had a much greater effect in the coastal site than in the desert. In the coastal site, this filter reduced species richness to $38.7 \%$ below the richness that would be expected if this site pool represented a random sample from the regional pool $(P<0.002$; Fig. 1$)$. The desert site, on the other hand, had only $4.5 \%$ fewer species than the random expectation, a non-significant difference $(P=0.084$; Fig. 1). We hypothesized that filtering at this scale would be due to limited long-distance dispersal or inappropriate climate. The coastal site, unlike the desert site was located at the edge of the Mediterranean Sea, and its edge location might limit long-distance dispersal. However, the desert site had the harsher average climate (Table 1), so average climate does not explain the stronger filter at the coastal site.

This filter may reflect climatic variability rather than average climate. Two additional analyses are consistent with the idea that greater climatic variability at the desert site might be responsible for maintenance of the higher diversity at this site. The coefficients of variation for monthly rainfall and minimum monthly temperature during the five growing seasons were greater in the desert than the coast (1.34 vs. 0.87 for rainfall, 0.58 vs. 0.51 for minimum temperature). Further, correspondence analysis revealed that species composition varied more among the five desert site-year pools (31\% of total variation among site-year pools) than among the five coastal site-year pools $(17 \%$ of total variation; the remaining variation is explained by site differences). Variations in climate may lead to variations in species composition and therefore greater long-term diversity in the desert.

The second filter, from site pools to site-year pools, significantly reduced species richness of each of the 10 siteyear pools from that expected based on random sampling from the site pools $(P<0.008)$ with the single exception of the coastal 1994 pool $(P=0.922)$. Although this filter was greater in the desert than the coast when averaged across years (Fig. 1), the contrast was not significant (ANOVA on lnRR, $F_{1,8}=1.646, P=0.235$ ) and the trend was only apparent in 3 of 5 years. We expected this filter to be caused by abiotic conditions, with species being eliminated in years with insufficient rainfall or inappropriate temperatures, or by species interactions, with species being eliminated in years when competition was intense. Species interactions did not seem to contribute to this filter. Competition is often reported as more intense in more productive communities, but plant production, measured as standing crop $\left(\mathrm{g} \mathrm{m}^{-2}\right)$ did not predict deviation from expected richness $\left(R^{2}=0.117\right.$, $\left.F_{1,8}=1.059, P=0.334\right)$. Furthermore, plot density varied three to 100 -fold in each site-year. The lowest-density plots should have low potential for species interactions; as long as poor competitors were able to persist in these plots, they should not have been eliminated from the entire site-year pool.

The second filter was associated with abiotic conditions, but not with the most obvious factor, rainfall. Annual rainfall, as well as mean and maximum temperatures, failed to predict deviation from expected richness among site-year pools (linear regression; yearly rainfall $R^{2}=0.104, F_{1,8}=$ $0.931, P=0.363$; mean temperature $R^{2}=0.112, F_{1,8}=$ 1.011, $P=0.344$; maximum temperature $R^{2}=0.010$, $\left.F_{1,8}=0.083, P=0.781\right)$. However, deviation from expected richness was predicted by minimum temperature (Fig. 2; linear regression, $\left.R^{2}=0.593, F_{1,8}=11.654, P=0.009\right)$. In each year, the desert site experienced more extreme minimum temperatures $\left(2.0-4.2^{\circ} \mathrm{C}\right)$ than the coastal site

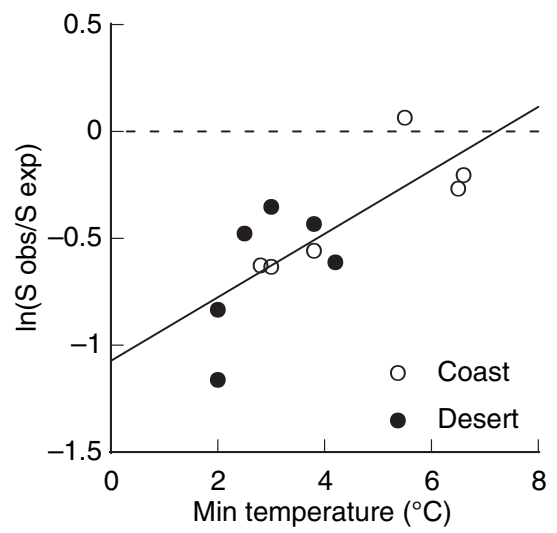

Figure 2 Deviation from expected richness (lnRR) as a function of minimum temperature during the season for each site-year pool. LnRR $<0$ indicates that ecological filters reduces richness to levels less than would be expected based on random sampling from the site pool. 


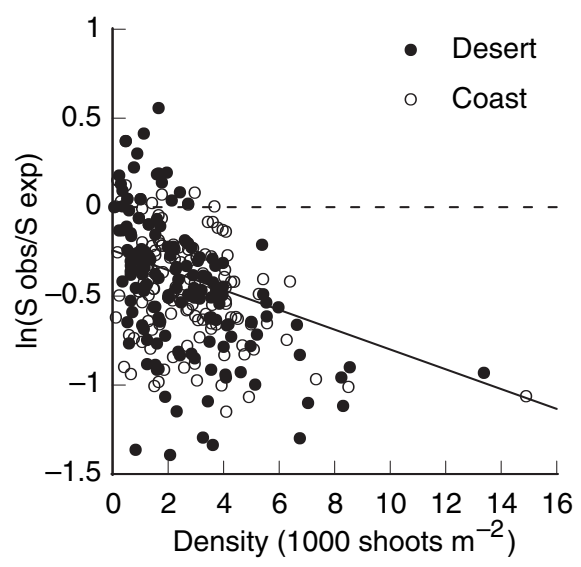

Figure 3 Deviation from expected richness (lnRR) as a function of local density of each plot. LnRR $<0$ indicates that ecological filters reduces richness to levels less than would be expected based on random sampling from the site-year pool. Regression results are similar when the two highest-density plots are removed from the analysis.

$\left(3.0-6.6^{\circ} \mathrm{C}\right)$, and lower minimum temperatures were associated with a larger decline of species richness in a site-year pool from that expected by randomly sampling a site pool (Fig. 2).

The next filter, from site-year pools to individual plots, again reduced species richness below that expected if plots represented random draws from the site-year pool, with the average plot having 30-35\% fewer species than the random expectation (Fig. 1). However, the strength of this filter was similar in the two communities (ANOVA on $\operatorname{lnRR}$; site $F_{1,273}=0.319, P=0.573$; site-year $F_{4,273}=$ 1.495, $P=0.204)$. In both communities, this filter was strongly density-dependent with the reduction of observed richness relative to that expected from random sampling of the pool greater at higher plot density (Fig. 3; three-way ANCOVA on lnRR; density effect $F_{1,263}=15.676, P<$ 0.001). This density-dependence suggests that the third filter was due to competition: plants in high-density plots competed more intensely, resulting in greater competitive exclusion than in low-density plots. This density effect did not significantly interact with site or year, despite the large differences in weather and productivity among sites and years.

\section{DISCUSSION}

The filter model, along with additional analyses, provides insight into the various factors that determine local, plotlevel species richness in our system. It also suggests that the processes that strongly influence the average local richness within a site are not necessarily the same processes that account for the differences in local, plot-level richness between sites.

The first filter, long-distance dispersal plus climate, had a much greater effect in the coastal site than in the desert (Fig. 1). This could be because the coastal site, which is near the Mediterranean Sea, represents an 'edge' location, and might experience less long-distance immigration of propagules from core populations. Alternatively, the greater variability in conditions at the desert site may contribute to maintaining more species in the overall site pool by favouring or suppressing different species in different years. Consistent with the latter explanation, monthly rainfall and minimum monthly temperature varied more during the five growing seasons in the desert than the coast, and species composition varied more among years in the desert than at the coast. Thus, the pattern of greater species density in the more variable desert site is consistent with a temporal mass effect (Shmida \& Ellner 1984) or storage effect (Chesson \& Warner 1981): different species dominate under different environmental conditions, but the presence of a persistent seed bank allows a few individuals of many species to emerge even under suboptimal conditions. This mechanism for increased diversity is highly plausible for desert annuals because species are well-known to differ in the temperature and rainfall conditions that trigger high germination rates (Shmida \& Ellner 1984; Gutterman 2002; Adondakis \& Venable 2004). Temporal mass effects are widely accepted as a plausible explanation for species coexistence, especially in annual communities with dormancy, but little empirical work has explicitly addressed their role in explaining diversity patterns between communities and the few examples that do address this issue are inconsistent. For example, one experimental study found that diversity in a grassland increased when temporal variability of rainfall was artificially increased (Knapp et al. 2002). In contrast, a survey of communities in Iberia found that local diversity decreased with increasing temporal heterogeneity of rainfall (Rey Benayas \& Scheiner 2002). The role of temporal storage effects in explaining between-community patterns in species diversity deserves further research. We unfortunately have data on only two sites; a more extensive data set would help confirm whether there is a consistent relationship between site-level filtering and climate variability. This question might also be further addressed in our existing data set by analyses that link species composition and changes in relative abundances, rather than just presence/absence, to rainfall or temperature.

The second filter, from site to site-year pools and potentially including reductions in richness due to abiotic stress or competition, reduced species richness in nine of the 10 site-year pools, but the reductions were similar in the two sites (Fig. 1). Therefore, we conclude that although this 
filter is substantial, it is similar between the two sites and cannot account for the difference in richness at the plot level between the sites. Competition likely contributed little to this whole-site level filter. Plant densities in individual plots varied three to 100 -fold within each site-year. As we do not have data on microenvironment of each plot and therefore the carrying capacity per plot is not known, localized dispersal probably plays a considerable role in this small-scale variation and therefore it is likely that competition was low in the lowest-density plots. Therefore, while competition may have reduced the abundance of poor competitors by eliminating them from high-density plots, we expect that few species were entirely eliminated from any site-year pool by competition.

We expected the abiotic filter to be driven by precipitation, because water and/or nutrients (with mineralization driven by water) are generally assumed to be limiting in sand dune communities, especially in the desert site. However, yearly rainfall at a site was a poor predictor of the deviation of observed richness in a site-year pool from expected. Instead, somewhat surprisingly, low temperatures appeared to be an important constraint: the siteyears with the lowest minimum temperature observed during the winter growing season had the greatest reduction from expected species richness (Fig. 2). Appropriate temperatures are an important requirement for seed germination, and cold temperatures may prevent germination of some species.

We initially suspected that either the first or second filter would reveal a productivity-diversity relationship between the two sites: that higher rainfall at the coast would lead to greater plant production which in turn produce a stronger filter on diversity, most likely through increased competition intensity (Grime 1977; Tilman 1982). If productivity was important, the same pattern might be expressed at a finer scale in the site-year pools, with higher productivity in wetter years leading to a stronger filter and lower diversity. Our results were not consistent with a strong direct effect of productivity on diversity: neither rainfall, expected to drive plant production, nor plant standing crop itself predicted deviation from expected richness. A previous study in similar sites also found no relationship between yearly rainfall and diversity within desert or coastal sites over 5 years, although there was a positive correlation at an intermediate site (Aronson \& Shmida 1992).

The third filter, potentially incorporating competitive exclusion at the plot scale, microenvironment effects, and local dispersal also reduced species richness by similar amounts in the two communities. Further, plots with the greatest density had the greatest decrease from expected richness within each site-year. These results are not consistent with a simple, direct effect of either microenvi- ronment or local dispersal. If density reflected favourability of the microenvironment, we would expect diversity to be limited less in higher-density plots (with more favourable microenvironment). Similarly, if plot-level richness was limited by local dispersal, we would expect less limitation in high-density plots, which had the greatest seed input. On the contrary, greater limitation of diversity in high-density plots is consistent with more intense competition in those plots. Therefore, we conclude that competition most likely drives the third filter. Intense competition may be attributable to high density alone (generated by random dispersal events). Alternatively, competition and microenvironment may interact to produce the density-dependence: productive sites are often associated with low diversity because competition is more intense there (Grime 1977; Tilman 1982).

Consistent with the interpretation that competition, rather than microenvironment, drives this filter, experimentally increasing density and therefore competition potential in garden plots significantly reduced richness in both communities relative to that expected (Goldberg \& Estabrook 1998), but experimental manipulations of rainfall did not (D.E. Goldberg, R. Turkington, A.R. Dyer, unpublished data). Microenvironmental variation would presumably be more subtle than the irrigation treatments, and therefore even less likely to affect species richness. Similarly, structural equation models have generally revealed strong negative effects of plant biomass or canopy light penetration, both assumed to be indicators of competition intensity, on local diversity (Grace \& Pugesek 1997; Grace \& Jutila 1999; Weiher 2003; Weiher et al. 2004).

The analyses presented here deal only with species richness. Our sampling method provides information on the effects of filters on abundance of individual species, as well as species richness. While these analyses are beyond the scope of the present study, they offer the potential to examine the processes influencing other aspects of community structure, such as other diversity indices, species composition or beta diversity among plots, years or sites.

\section{CONCLUSIONS}

Processes operating at both regional and local scales and within and among years all reduce local species richness relative to that expected by chance in these two annual plant communities (Fig. 1). Further, on average across the two sites, processes working on several different spatial and temporal scales have similar magnitudes of effect on richness. However, only the largest scale processes that resulted in a smaller site pool at the coast explain the typically lower plot-level richness in that community. 
More generally, we suggest that such a pattern, where the relative importance of different processes accounting for small-scale species richness within a site does not necessary correspond to the importance of those processes in explaining patterns in species richness between sites, may indeed be very common. However, we need many more examples of quantitative partitioning of the processes that control species diversity, rather than simply testing for signals of any influence of a process, before we can begin to understand the relative importance of ecological processes for explaining aspects of community structure within versus between communities. The quantitative filter approach we employ in this study is a relatively simple way to generate such information from existing data sets.

\section{ACKNOWLEDGEMENTS}

Too many people to name individually helped in collecting and processing vegetation samples over the years; we thank them all. Zuzana Munzbergova and Vigdis Vandvik helped with data analysis and they, Earl Werner, Martin Zobel, Jim Grace and two anonymous referees provided helpful comments on previous drafts of the manuscript. The US-Israel Binational Science Foundation, NSF and NSERC provided funding. This is publication no. 504 of the Mitrani Department of Desert Ecology, Blaustein Institute of Desert Research, Ben Gurion University, Israel.

\section{REFERENCES}

Adondakis, S. \& Venable, D.L. (2004). Dormancy and germination in a guild of Sonoran Desert annuals. Ecology, 85, 25822590.

Aronson, J. \& Shmida, A. (1992). Plant species diversity along a Mediterranean-desert gradient and its correlation with interannual rainfall fluctuations. J. Arid Environ., 23, 235-247.

Chesson, P.L. \& Warner, R.R. (1981). Environmental variability promotes coexistence in lottery competitive systems. Am. Nat., 117, 923-943.

Foster, B.L. \& Dickson, T.L. (2004). Grassland diversity and productivity: the interplay of resource availability and propagule pools. Ecology, 85, 1541-1547.

Foster, B.L., Dickson, T.L., Murphy, C.A., Karel, I.S. \& Smith, V.H. (2004). Propagule pools mediate community assembly and diversity-ecosystem regulation along a grassland productivity gradient. J. Ecol., 92, 435-449.

Goldberg, D.E. \& Estabrook, G.F. (1998). Separating the effects of number of individuals sampled and competition on species diversity: an experimental and analytic approach. J. Ecol., 86, 983-988.

Goldberg, D.E., Turkington, R., Olsvig-Whittaker, L. \& Dyer, A.R. (2001). Density dependence in an annual plant community: variation among life history stages. Ecol. Monogr., 71, 423446.
Gough, L., Grace, J.B. \& Taylor, K.L. (1994). The relationship between species richness and community biomass: the importance of environmental variables. Oikos, 70, 271-279.

Grace, J.B. (2001). Difficulties with estimating and interpreting species pools and the implications for understanding patterns of diversity. Folia Geobot., 36, 71-83.

Grace, J.B. \& Jutila, H. (1999). The relationship between species density and community biomass in grazed and ungrazed coastal meadows. Oikos, 85, 398-408.

Grace, J.B. \& Pugesek, B.H. (1997). A structural equation model of plant species richness and its application to a coastal wetland. Am. Nat., 149, 436-460.

Grime, J.P. (1977). Evidence for existence of three primary strategies in plants and its relevance to ecological and evolutionary theory. Am. Nat., 111, 1169-1194.

Gutterman, Y. (2002). Minireview: survival adaptations and strategies of annuals occurring in the Judean and Negev Deserts of Israel. Isr. J. Plant Sci., 50, 165-175.

Keddy, P.A. (1992). Assembly and response rules: two goals for predictive community ecology. J. Veg. Sci., 3, 157-164.

Knapp, A.K., Fay, P.A., Blair, J.M., Collins, S.L., Smith, M.D., Carlisle, J.D. et al. (2002). Rainfall variability, carbon cycling, and plant species diversity in a mesic grassland. Science, 298, 22022205.

Mouquet, N., Leadley, P., Meriguet, J. \& Loreau, M. (2004). Immigration and local competition in herbaceous plant communities: a three-year seed-sowing experiment. Oikos, 104, $77-90$.

Partel, M., Zobel, M., Zobel, K. \& van der Maarel, E. (1996). The species pool and its relation to species richness: evidence from Estonian plant communities. Oikos, 75, 111-117.

Rey Benayas, J.M. \& Scheiner, S.M. (2002). Plant diversity, biogeography and environment in Iberia: patterns and possible causal factors. J. Veg. Sci., 13, 245-258.

Ricklefs, R.E. (2004). A comprehensive framework for global patterns in biodiversity. Ecol. Lett., 7, 1-15.

Safford, H.D., Rejmanek, M. \& Hadac, E. (2001). Species pools and the "hump-back" model of plant species diversity: an empirical analysis at a relevant spatial scale. Oikos, 95, 282-290.

Shmida, A. \& Ellner, S. (1984). Coexistence of plant species with similar niches. Vegetatio, 58, 29-55.

Tilman, D. (1982). Resource Competition and Community Structure. Princeton University Press, Princeton, NJ.

Tilman, D. (1997). Community invasibility, recruitment limitation, and grassland biodiversity. Ecology, 78, 81-92.

Weiher, E. (2003). Species richness along multiple gradients: testing a general multivariate model in oak savannas. Oikos, 101, 311316.

Weiher, E., Forbes, S., Schauwecker, T. \& Grace, J.B. (2004). Multivariate control of plant species richness and community biomass in blackland prairie. Oikos, 106, 151-157.

Xiong, S., Johansson, M.E., Hughes, F.M.R., Hayes, A., Richards, K.S. \& Nilsson, C. (2003). Interactive effects of soil moisture, vegetation canopy, plant litter and seed addition on plant diversity in a wetland community. J. Ecol., 91, 976-986.

Zobel, M. (1992). Plant species coexistence - the role of historical, evolutionary and ecological factors. Oikos, 65, 314-320.

Zobel, M. (1997). The relative role of species pools in determining plant species richness: an alternative explanation of species coexistence? Trends Ecol. Evol., 12, 266-269. 
Zobel, M., van der Maarel, E. \& Dupre, C. (1998). Species pool: the concept, its determination and significance for community restoration. Appl. Veg. Sci., 1, 55-66.

Zobel, M., Otsus, M., Liira, J., Moora, M. \& Mols, T. (2000). Is small-scale species richness limited by seed availability or microsite availability? Ecology, 81, 3274-3282.

Zohary, M. (1966). Flora Palaestina. Israel Academy of Sciences and Humanities, Jerusalem.
Editor, James B. Grace

Manuscript received 9 May 2005

First decision made 16 June 2005

Second decision made 17 August 2005

Manuscript accepted 6 October 2005 